\title{
Pharmacological, Technological, and Digital Innovative Aspects in Rhinology
}

\begin{abstract}
Rosanna Ruggiero ${ }^{1,2 *}$, Giovanni Motta ${ }^{3}$, Giuseppe Massaro ${ }^{3}$, Concetta Rafaniello ${ }^{1,2}$, Alberto Della Corte ${ }^{2}$, Antonella De Angelis ${ }^{2}$, Annalisa Capuano ${ }^{1,2}$, Gaetano Motta ${ }^{3+}$ and Francesco Rossi ${ }^{1,2+}$

${ }^{1}$ Campania Regional Centre for Pharmacovigilance and Pharmacoepidemiology, Naples, Italy, ${ }^{2}$ Department of Experimental Medicine, Section of Pharmacology "L. Donatelli", University of Campania "Luigi Vanvitelli", Naples, Italy, ${ }^{3}$ Department of Mental Health and Public Medicine, Section of Otorhinolaryngology - Head and Neck Surgery, University of Campania "Luigi Vanvitelli", Naples, Italy
\end{abstract}

Innovation refers to the introduction of a product, a process, a service or a solution resulting in something new or significantly improved compared to the already available alternatives. In the clinical context, it is strictly related to the identification of a new added value in terms of quality, therapeutic efficacy and safety. Over the years several innovative approaches have been introduced in the clinical practice, revolutionizing the treatment and the management of important rhinologic conditions. Innovative tools, including new drugs, biomaterials, and mobile applications seem to be able to improve the clinical outcomes and the quality of life of many patients affected by (often relapsing) rhinologic diseases. Among the main modern pharmacological innovations, mention must be made of the biological drugs like monoclonal antibodies (mAbs). Recently, new mAbs have been introduced and investigated as useful arms in the treatment of some inflammatory/infectious or oncological diseases affecting the nasal cavities and paranasal sinuses. The already approved or still investigated mAbs work inhibiting different type 2 inflammation pathways, including those mediated by lgE (omalizumab), IL-4/IL-13 (dupilumab), and IL-5 (mepolizumab). Moreover, considering the higher expression of PD-L1 in nasopharyngeal carcinoma, the use of PD-1 inhibitors, such as nivolumab, or a dual CTLA-4/PD-1 blockade (ipilimumab plus nivolumab) appear to be an effective strategy for the treatment of this cancer form. The implants with bio-absorbable biomaterials represent new interesting available technological innovations. Moreover, advanced technologies such as the artificial intelligence, the machine learning as well as the augmented or virtual reality have also proved useful in rhinologic field with main impacts on precision medicine and surgery. Finally, the development and use of mobile-Health tools represent a winning strategy in monitoring of the therapy success, safety and tolerability as well as the progress of chronic disease including chronic rhinosinusitis with nasal polyps. Supporting the research of innovative tools and strategies (including pharmacological, technologic, or digital ones) is essential to improve the management of chronic diseases that significantly affect the patients' quality of life.

Keywords: pharmacological innovation, digital innovation, technological innovation, monoclonal antibodies, dupilumab, mepolizumab, chronic rhinosinusitis (CRS), rhinology 


\section{INTRODUCTION}

Innovation refers to the introduction of a product, a process, a service or a solution resulting in something new or significantly improved compared to the already available alternatives (1). Regarding drugs, innovation is strictly related to the identification of an added value in terms of quality, therapeutic efficacy and safety, established on the basis of the results emerging from randomized clinical trials. The research and development of innovative medicines is fundamental to address persisting unmet therapeutic needs. Both the Food and Drug Administration (FDA) and the European Medicines Agency (EMA) support it through the so-called Early Access Programs (EAPs) (2). In Italy, the marketing authorization of a medicinal product, the possible recognition of its reimbursement or its innovativeness are not automatically consequential. Even if substantially based on the same evidence, they represent three distinct procedures. Using a multidimensional approach, the therapeutic need, the added therapeutic value and the quality of evidence represent the three variables to consider in the innovative evaluation (3).

Current innovations can involve three overlapping domains: pharmacological, referred to the discovery of new molecules with innovative mechanisms of action or the recognition of new therapeutic indications for already authorized drugs, technological considering new release/administration systems of already available drugs, and digital such as new medical software or applications. Today, we are experiencing a renaissance of innovation. Recently, several innovative drugs have been approved and introduced into clinical practice, revolutionizing the treatment of important diseases, such as hepatitis $\mathrm{C}$ or several types of cancer. Still other new innovative drugs are going to be authorized (new monoclonal antibodies for the treatment of Alzheimer's, neoplasms, asthma, chronic obstructive pulmonary disease and cardiovascular diseases). Pharmaceutical innovation allowed important therapeutic results. Thanks to innovative drugs, it has been possible to increase the life expectancy of many patients, transforming lethal pathologies into chronic ones. The increase in the 5-year mortality rate for various oncological diseases as well as the reduction in the mortality rate of HIV/AIDS are unequivocal examples. Likewise, innovative drugs are enabling continued advances in the management of the COVID-19 pandemic. Such progress is also made possible by the introduction of innovative research models. Over the years, the Research and Development sectors of biopharmaceutical companies evolved from a closed innovation model, where innovation was centralized within the company, to arrive at an open innovation enabling collaborations outside the company. Today, the companies are increasingly concentrated in network innovation activities, i.e., the acquisition of research and development services ( $R \& D$ extra muros), machinery and software aimed at innovation and skills from other companies or institutions (4).

Pharmacological innovation has also involved the field of rhinology. A variety of conditions affect the nose and sinuses, including inflammatory diseases, i.e., rhinitis, sinusitis, nasal polyposis, up to tumors of the nasal cavities and paranasal sinuses (Figure 1). The traditional treatments for inflammatory rhinological diseases include symptomatic therapies, based on antihistamine drugs and nasal decongestants, and disease modifying treatments, such as topical corticosteroids. New innovative drugs are able to improve clinical outcomes and quality of life of many patients affected by (often relapsing) rhinological diseases. Some of the main advances achieved in terms of pharmacological, technological as well as digital innovation applied to the field of rhinologic diseases are described below.

\section{PHARMACOLOGICAL INNOVATION}

Among the main modern pharmacological innovations, biological drugs are revolutionizing the treatment of several pathologies, finding application in many therapeutic fields, including the rhinologic one (5). A biological drug is characterized by an active substance (generally a high molecular weight protein) produced by a living organism (microorganisms or animal cells) or using a biological source through the use of recombinant DNA techniques (biotechnological drugs). Biologics are more complex molecules than chemical drugs. Their major complexity is associated with an increase in their structural dimensions. Among the main classes or categories of biologicals, monoclonal antibodies $(\mathrm{mAb})$ are worthy of note (6). Over the years, the approved $\mathrm{mAb}$ therapies have seen incredible growth, evidenced by the fact that in 2018, globally, six out of 10 best-selling drugs were mAbs (7). Today, in the COVID-19 pandemic context, mAbs represent an important part of the therapeutic armamentarium useful against SARSCoV-2. Since they are able to block the viral attachment of SARS-CoV-2 to host cells, they seem to be promising tools in patients at early stage of COVID-19, preventing its progression and reducing the morbidity and mortality of infection such as the frequency of hospitalizations $(8,9)$. Overall, excellent efficacy profiles and lower frequency of adverse reactions characterize these drugs. Recently, some mAbs have been introduced and investigated as useful arms in the treatment of some inflammatory/infectious or oncological diseases affecting the nasal cavities and paranasal sinuses.

\section{Innovative Drugs for the Treatment of Inflammatory/Infectious Rhinological Conditions}

The rhinological diseases sharing inflammatory features, such as airway eosinophilia, local IgE formation, and a $\mathrm{TH}_{2}$ cytokine profiles, are evaluated as possible indications for some mAbs (10). Nasal polyps (NP), asthma, rhinitis and sinusitis, individually and in their various possible associations, represent some of these clinical challenges. Moreover, these pathologic conditions are often comorbid, with serious effect on the quality of life of patients. The already approved or still investigated mAbs work inhibiting different type 2 inflammation pathways, including those mediated by IgE, IL-4, IL-5, and IL-13 (Figure 2). Such mAbs represent useful tools for a precision medicine approach in the evaluation and management of severe chronic inflammatory conditions of upper respiratory tract (11), such 


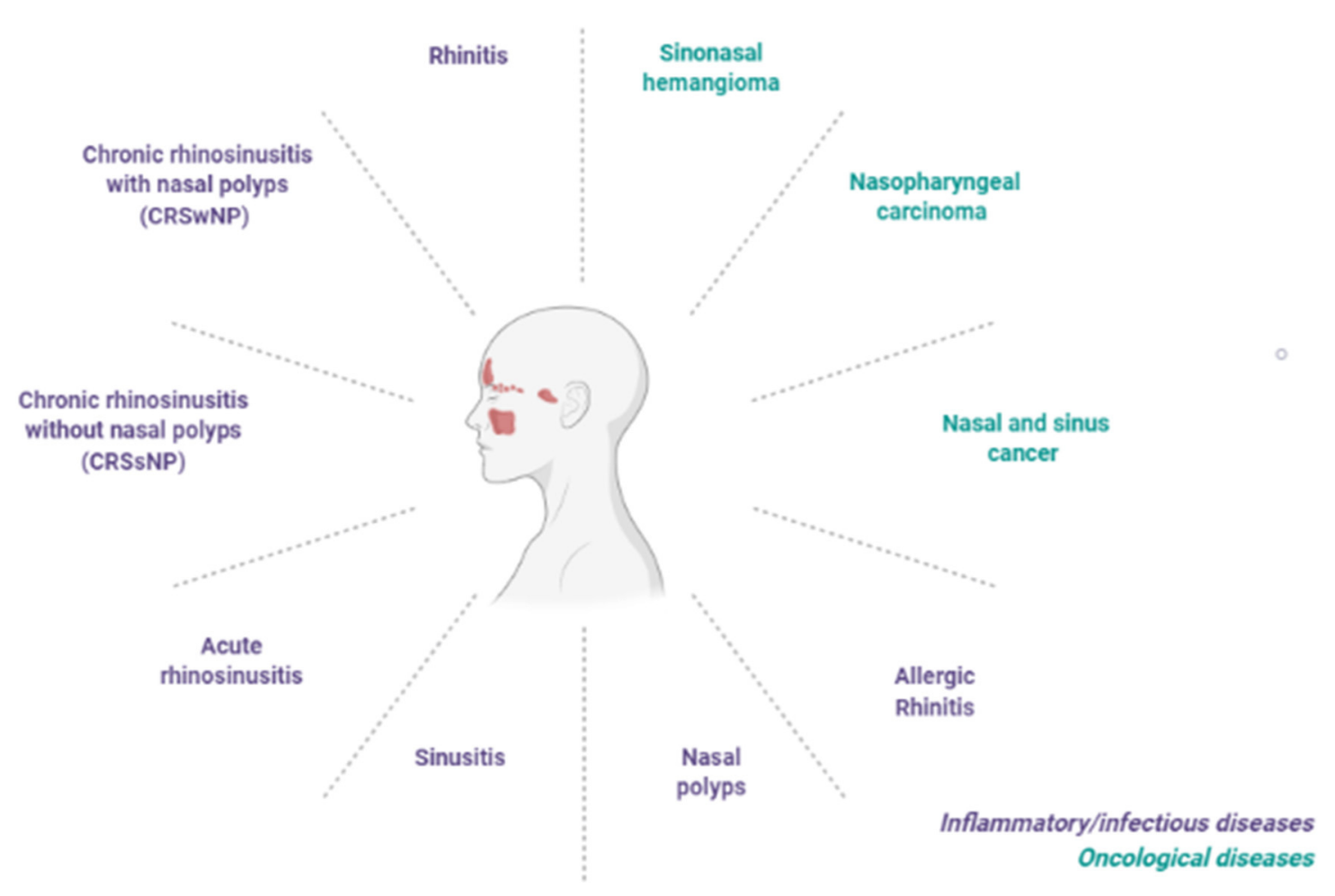

FIGURE 1 | Main inflammatory/infective and oncological conditions affecting the nose and sinus cavities or upper respiratory tract.

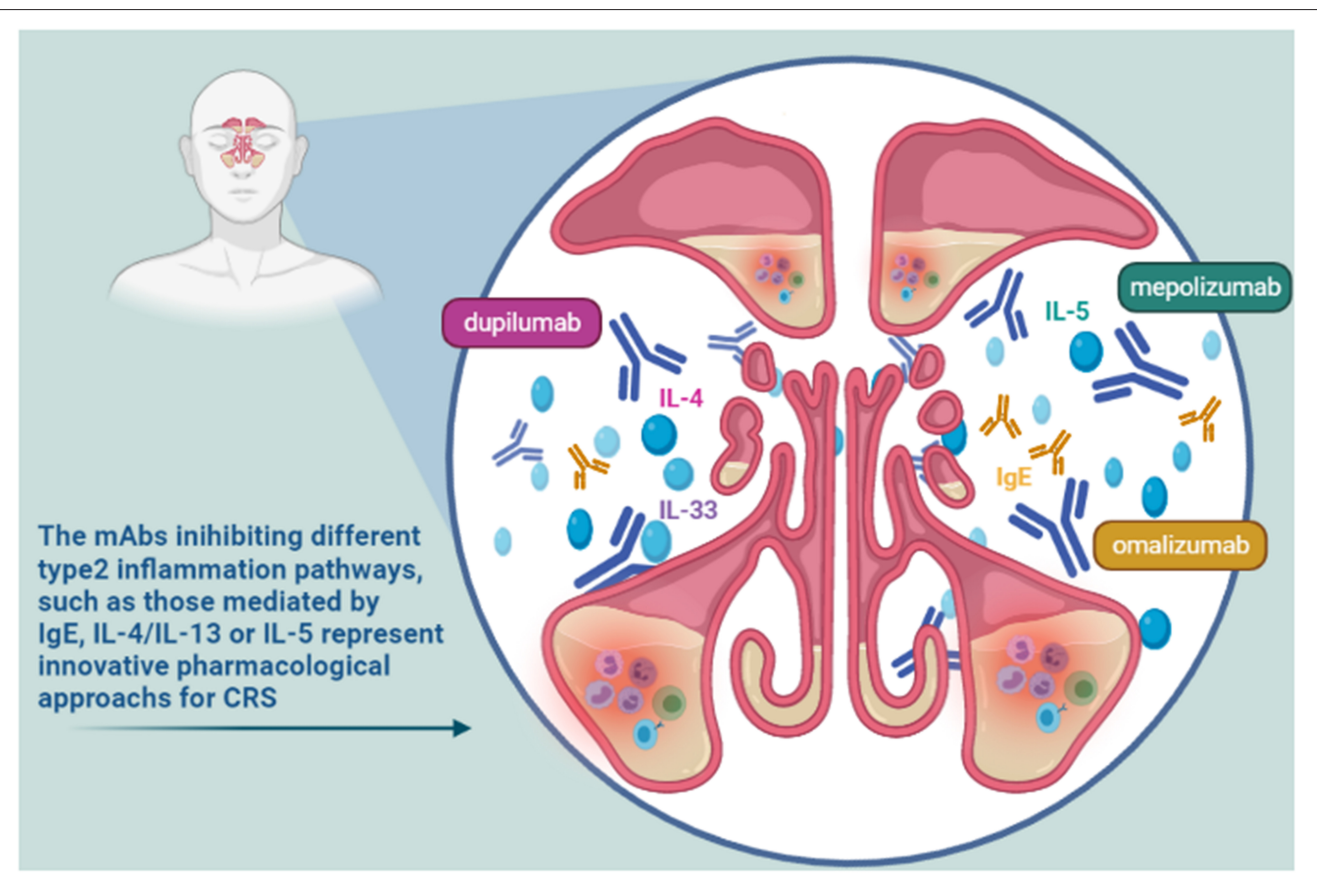

FIGURE 2 | Some target pathways target of mAbs effective in chronic rhinosinusitis (CRS).

as chronic rhinosinusitis (CRS) $(11,12)$. CRS is characterized by local inflammation of the upper airways and sinuses which persists for at least 12 weeks (13). It affects $\sim 3 \%$ of the population worldwide (14). It is often associated with several co-morbidities including nasal polyps, asthma, acute infection, and obstructive sleep apnea. Based on the associated presence 
of nasal polyps, CRS was classified into two phenotypes: CRS with nasal polyps (CRSwNP) and CRS without nasal polyps (CRSsNP) (15). The investigated mAbs seem to be particularly effective in the management of CRSwNP. This phenotype is predominantly an adult disease, with an average onset between 40 and 60 years old, frequently associated with severe asthma. It is difficult to treat, as often relapsing, even after surgery. CRSwNP is a debilitating disease accompanied by complete anosmia, headaches, often requiring chronic therapies with douching, topical corticosteroids, systemic corticosteroids and antibiotics, plus repeated surgical polypectomies to control the disease (16). This disease shows a substantial clinical and economic burden, significantly impacting on patients' lives and often causing missed work, and hospitalizations (17). Most patients affected by CRSwNP show a type 2 inflammatory form in the nasal and paranasal sinus mucosa. In particular, the degree of type 2 inflammation is correlated with disease severity of CRSwNP. On the other hand, in about $80 \%$ of patients chronic rhinosinusitis is characterized by the absence of nasal polyps. This disease phenotype is primarily associated to type 1 inflammation (13). Neverthless, increased levels of IL-4, IL-5, and IgE have been recently observed also in some patients with CRSsNP. So, some mAbs targeting on these pathways might be effective also in this patients population (18). In the light of this recent evidence, in the European Position Paper on Rhinosinusitis and Nasal Polyps (EPOS) 2020 the previous phenotype-based classification of CRS has been replaced, highlighting the anatomic distribution (localized or diffuse) and endotype dominance (type 2 or non-type 2) (19). To date, IgE, IL-4, and IL-5 represent the main targets of identified effective mAbs. Moreover, other possible targets for biological treatment of eosinophil and mast cell-related diseases such as CRSwNP seem to be IL-33, IL17, thymic stromal lymphopoietin (TSLP) (Table 1). Overall, biologic therapy with mAbs targeting IgE (omalizumab), IL$4 \mathrm{R} \alpha$ (dupilumab), or IL-5 (reslizumab, mepolizumab) led to the improvement of several clinical outcomes, including reduction size of nasal polyps, favorable impact on quality of life, nasal airflow capacity and smell. Overall, the use of these agents was found to be safe and well-tolerated (20). Recently, a Cochrane Review focusing on the clinical management of patients with NP and CRS evaluated studies referred to three main biologics dupilumab, mepolizumab, and omalizumab. Disease-specific health-related quality of life (HRQL), disease severity and serious adverse events were the primary outcomes. All the patients enrolled in the included studies were using topical nasal steroids. According to the results (summarized in Table 2), dupilumab represents the $\mathrm{mAb}$ inducing more improvement in all considered primary outcomes (16).

\section{mAbs Targeting the IgE Pathway}

Omalizumab is an anti-IgE humanized mAb produced by recombinant DNA technology. It was already approved in 2002 for the treatment of allergic asthma in adults, adolescents and children aged $\geq 6$ years. Since February 2014, it was also approved for the treatment of chronic spontaneous urticaria in patients aged $\geq 12$ years. Finally, in July 2020 , omalizumab obtained another extension of indication as add-on therapy for the treatment of adults (age $\geq 18$ years) with severe CRSwNP for whom only intranasal corticosteroid therapy does not provide adequate disease control (21). Omalizumab dosing reflects the personalized approach to which biological therapy is aimed. In fact, as reported in the Summary of Product Characteristics, it is determined considering the baseline serum IgE level $(\mathrm{UI} / \mathrm{mL})$ and body weight $(\mathrm{Kg})$ of each patient. Based on these determinations, the dosing (from 75 to $600 \mathrm{mg}$ ) and the time intervals for its subcutaneous administration (every 2 or 4 weeks) are identified ${ }^{1}$. Binding selectively to IgE, omalizumab reduces the concentration of free $\mathrm{IgE}$ in blood and in tissue, surface $\operatorname{IgE}$ on basophils and mast cells and, consequently, blocks the effects of IgE on dendritic cells ${ }^{1}$. IgE is involved in some biological functions and mechanisms relevant for several diseases, including allergic rhinitis and nasal polyposis. Overall, omalizumab treatment is able to induce a reduction of nasal polyp size, an improvement of symptoms and the inhibition of underlying type 2 inflammation ${ }^{2}$. Several randomized, doubleblind, placebo-controlled trials such as real-life studies showed omalizumab efficacy in the disturbances of nasal and/or sinusal mucosa. In particular, the results of a randomized, doubleblind, placebo-controlled study showed omalizumab efficacy in improving airway symptoms (including nasal congestion, anterior rhinorrhea, loss of sense of smell, wheezing, and dyspnea) and quality-of-life scores in patients with nasal polyps and comorbid asthma. Its clinical efficacy occurred irrespective of the presence of allergy (10). Moreover, according results of a recent real-life study, omalizumab was able to treat both CRSwNP and asthma. The induced improvements in CRSwNP control were rapid and similar to that obtained with upper airway surgery (17). Finally, a recent study aimed to analyse in a real-life setting the therapeutic outcomes of $\mathrm{mAb}$ treatments, including omalizumab, was published. Moreover, the authors tried to identify possible predictive biomarkers for successful therapy. Their results confirmed the biologicals as promising treatment option of CRSwNP, especially in severe cases not responding to conventional therapy (22). Ligelizumab and quilizumab are other anti-IgE mAbs, mainly investigated as treatments for chronic spontaneous urticaria. Actually no trials for ligelizumab in rhinologic diseases have been yet initiated, while the evaluation of quilizumab efficacy in patients with allergic rhinitis is still in its early stages (Table 1) (23).

\section{mAbs Targeting the IL-5 Pathway}

IL-5 is another key driver of local type2-inflammation, produced by Th2 cells and group 2 innate lymphoid cells (ILC2s), stimulating the production, activation and maturation of eosinophils $(24,25)$. Approximately $85 \%$ of nasal polyps (NPs) are characterized by prominent eosinophilia. So, IL-5 inhibition with specific $\mathrm{mAbs}$ represent an innovative therapeutic approach in patients with NP or CRSwNP (26). To date, three mAbs targeting IL-5 (mepolizumab and reslizumab) or $\alpha$-subunit of its receptor (benralizumab) have been developed for clinical

\footnotetext{
${ }^{1}$ CHMP. Annex I Summary of Product Characteristics.

${ }^{2}$ Omalizumab (2021). Available online at: http://www.ncbi.nlm.nih.gov/pubmed/ 30000860 (accessed April 23, 2021).
} 
TABLE 1 | Main pathways target of recent innovative pharmacological treatments, their approved indications in Europe and United States and study phase for other inflammatory/infective sinonasal conditions.

\begin{tabular}{|c|c|c|c|c|c|}
\hline Pathways & mAbs & Target & $\begin{array}{l}\text { Approved indications in } \\
\text { EU by ema }\end{array}$ & $\begin{array}{l}\text { Approved indications in } \\
\text { us by FDA }\end{array}$ & $\begin{array}{l}\text { studies phase in NP, CRS, } \\
\text { or CRSwNP, CRSsNP, AR }\end{array}$ \\
\hline \multirow[t]{3}{*}{$\lg E$} & Omalizumab & $\lg E$ & $\begin{array}{l}\text { - CRSwNP } \\
\text { - CIU } \\
\text { - Allergic asthma }\end{array}$ & $\begin{array}{l}\text { - Nasal polyps } \\
\text { - CIU } \\
\text { - Allergic asthma }\end{array}$ & Phase 3 (AR) \\
\hline & Ligelizumab & $\lg E$ & - & - & No trials yet \\
\hline & Quilizumab & $\lg E$ & - & - & Phase 1 (AR) \\
\hline \multirow{2}{*}{ IL-5 } & Reslizumab & $\mathrm{IL}-5$ & - Eosinophilic asthma & - Eosinophilic asthma & Phase 3 (CRS) \\
\hline & Benralizumab & $\mathbb{I L}-5 \mathrm{R} \alpha$ & - Eosinophilic asthma & - Eosinophilic asthma & $\begin{array}{l}\text { Phase } 3 \text { (NP) Phase } 3 \\
\text { (CRSwNP) }\end{array}$ \\
\hline IL-4/IL-13 & Dupilumab & $\mathrm{IL}-4 \mathrm{R} \alpha$ & $\begin{array}{l}\text { - Atopic dermatitis } \\
\text { - Asthma } \\
\text { - CRSWNP }\end{array}$ & $\begin{array}{l}\text { - Atopic dermatitis } \\
\text { - Asthma } \\
\text { - CRSWNP }\end{array}$ & $\begin{array}{l}\text { Phase } 3 \text { (CRSnNP) Phase } 4 \\
\text { (CRSwNP) }\end{array}$ \\
\hline $\mathrm{IL}-17$ & Brodalumab & IL-17RA & - Psoriasis & - Psoriasis & No trials yet \\
\hline IL-33 & Etokimab & IL-33 & - & - & Phase 2 (CRSwNP) \\
\hline TSLP & Tezepelumab & TSLP & - & - & Phase 3 (CRSwNP) \\
\hline
\end{tabular}

CIU, chronic idiopathic urticarial; AR, Allergic Rhinitis; NP, nasal polyps; TSLP, thymic stromal lymphopoietin; EGPA, eosinophilic granulomatosis with polyangiitis; HES, hypereosinophilic syndrome; CRS, chronic rhinosinusitis; CRSSNP, chronic rhinosinusitis without nasal polyps; CRSwNP, chronic rhinosinusitis with nasal polyps, EU, Europe; US, United States; EMA, European Medicines Agency; FDA, Food and Drugs Administration.

TABLE 2 | Main results of a recent Cochrane Review on the clinical management of patients with NP and CR with biologics.

\begin{tabular}{llll}
\hline mAb compared to placebo & Disease-specific HRQL & Disease severity & Serious adverse events \\
\hline Dupilumab (anti IL-4) & Improve & Results in a reduction & May result in a reduction in number \\
Mepolizumab (anti IL-5) & May improve & Very uncertain difference & Very uncertain difference \\
Omalizumab (antilgE) & Probably improve & No evidence & Very uncertain difference \\
\hline
\end{tabular}

use. Considering the important role of IL-5 in the development of bronchial hyper-responsiveness, all three mAbs have been evaluated in large-scale clinical trials as treatment for severe asthma. However, major studies evaluating the efficacy in patients with NP and CRSwNP have been conducted for mepolizumab. According the results of a meta-analysis, anti-IL5 therapy with mepolizumab induces a reduction in nasal polyp score in patients with CRSwNP (27).

Mepolizumab is a humanized $\mathrm{mAb}$ that binds IL-5, preventing its interaction with the $\alpha$-chain of the IL- 5 receptor (IL-5R $\alpha$ ). It was authorized by the EMA and the FDA in 2015 as an add-on treatment for asthma (28). Mepolizumab is innovative also for its pharmaceutical form of pre-filled syringe or prefilled pen authorized by the EMA in 2019 representing the first European biologic drug for which self-administration in severe eosinophilic asthma was possible (29). In 2020, the regulatory approval for new additional indications for mepolizumab was submitted to EMA. These included three other eosinophil-driven diseases such as CRSwNP, hypereosinophilic syndrome (HES), and eosinophilic granulomatosis with polyangiitis (EGPA) (29). In the United States, mepolizumab has been already approved as treatment for adult patients with EGPA and represents the first and only biologic treatment for HES approved by the FDA. Moreover, it still waiting for FDA authorization as treatment for CRSwNP. Mepolizumab efficacy in nasal or sinus disturbances has been investigated in several studies. Already in a first randomized, double-blind, placebo-controlled study emerged that mepolizumab reduced the need for surgery at Week 25 and induced a greater improvement in symptoms compared to placebo. For this study patients were enrolled with recurrent eosinophilic nasal polyposis receiving topical corticosteroids and who required surgery. Mepolizumab's efficacy was accompanied with a safety profile comparable with placebo (30). Moreover, the efficacy and safety of mepolizumab as treatment of recurrent, refractory severe bilateral CRSwNP in adult patients was assessed in the SYNAPSE study, a multicentric randomized, doubleblind, placebo-controlled, parallel-group, and phase 3 trial (31). According to the results of this study, mepolizumab represents an effective add-on treatment option to standard of care for CRSwNP. In particular, 414 patients enrolled in this study were randomly assigned (1:1) to receive mepolizumab subcutaneously $(100 \mathrm{mg})$ or placebo once every 4 weeks in addition to standard 
of care (mometasone furoate intranasal spray, saline nasal irrigations, systemic corticosteroids or antibiotics, or both), as required. At week 52 from baseline, endoscopic nasal polyp score and nasal obstruction VAS score were significantly improved in the mepolizumab group compared to the placebo one.

Reslizumab is another humanized monoclonal antibody approved in Europe and the USA for adult patients as add-on maintenance treatment for severe asthma with an eosinophilic phenotype (32). Reslizumab binds IL-5 with a picomolar affinity, reducing consequently survival and activity of eosinophils (33). Regarding its efficacy in nasal or sinus mucosal diseases, few data are available. In 2016, a first double blind, randomized, placebo-controlled, phase III trial started in California with the purpose of determinating whether reslizumab treatment was effective also for the chronic sinusitis. To date, although the study has passed its completion date, its status on Clinicaltrial.gov database results unknown (34). Another study evaluating the efficacy for the chronic rhinosinusitis symptoms in asthma patients undergoing reslizumab treatment was conducted in United States by the Department of Otolaryngology Head and Neck Surgery, University of Rochester. Its primary objective was to monitor the CRS symptoms in this patient population. This was a prospective observational study started in 2017, but subsequently withdrawn (34). Finally, a third mAb targeting on anti-IL5 pathways, benralizumab, is actually authorized in Europe as an add-on treatment in adults with eosinophilic asthma inadequately controlled despite high-dose inhaled corticosteroids plus long-acting $\beta$-agonists. This humanized monoclonal antibody targets IL-5R $\alpha$ with high affinity and specificity. The IL-5 receptor is specifically expressed on the surface of eosinophils and basophils. Benralizumab reduces eosinophilic inflammation by inducing the apoptotic process of eosinophils and basophils, through enhanced antibodydependent cell-mediated cytotoxicity (ADCC) ${ }^{3}$. Recently, a subanalysis of the Phase IIIb ANDHI trial has been published, whose results extend benralizumab's efficacy to severe eosinophilic asthma patients with comorbid NP (any severity) (35). In particular, improvements in the annualized Sino-Nasal Outcome Test-22 (SNOT-22), asthma exacerbation rate (AER), FEV 1, Asthma Control Questionnaire 6 (ACQ-6), and St. George's Respiratory Questionnaire (SGRQ) total score were observed with benralizumab treatment compared to placebo. Likewise, benralizumab efficacy and safety profile in patients with severe NP was confirmed by the results of another randomized, double-blind, placebo-controlled trial conducted by Tversky et al. For this study, 24 patients with severe NP (defined by endoscopic grade 5 or more out of 8) and elevated eosinophils, with a history of previous surgical or endoscopic polypectomy, were enrolled. Benralizumab achieved a statistically significant reduction in nasal polyp size, sinus occupancy, symptoms and improved sensation of smell for $83 \%$ of patients. Moreover, it was well-tolerated (36). Recently, Humanitas Clinical and Research of Rozzano Hospital (Milan, Italy) conducted a pilot,

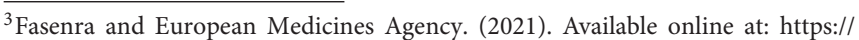
www.ema.europa.eu/en/medicines/human/EPAR/fasenra\#product-informationsection (accessed April 30, 2021).
}

prospective, double-blind, placebo-controlled, phase III-b trial in order to assess benralizumab clinical efficacy after week 24 of treatment. For this study, benralizumab $30 \mathrm{mg}$ was subcutaneously administered in patients with CRSwNP (allergic and non-allergic), every 4 weeks for the first 3 doses and then every 8 weeks. Moreover, in order to identify any possible predictive biomarker of response, an inflammatory e molecular phenotyping of responders to benralizumab was performed. Its results have been included in the aforementioned Cochrane Reviews, Biologics for chronic rhinosinusitis (16).

\section{mAbs Targeting the IL-4/IL-13 Pathway}

IL-4 and IL-13 are two Th2-associated cytokines with a mutual and important role in the type 2 inflammation. They share a same heterodimeric receptor, consisting in the combination of two subunits, IL- $13 \mathrm{R} \alpha 1$ and IL- $4 \mathrm{R} \alpha$ chain. This can be activated by both IL-4 and IL-13 (13). In particular, IL-4 and IL-13 pathways induce effects on keratinocytes (impairing their differentiation), eosinophils (inducing their activation), fibroblasts (increasing the production of eotaxin), B cells (IgE production), Th2 cells (increased the differentiation and survival). They play a fundamental role in the pathogenesis of nasal polyposis. Dupilumab, binding to IL-4R $\alpha$, blocks signaling of both the IL- 4 and IL-13 pathways, resulting in a powerful inhibition of Th2, eosinophil recruitment, and IgE production. Dupilumab is a completely human $\mathrm{mAb}$, administered as a subcutaneous injection every 2 weeks. Initially dupilumab has been authorized for the treatment of asthma and atopic dermatitis, as Th2 mediated diseases. Subsequently, it was also approved, first by the FDA (in June 2019) and then by the EMA (November 2019), as the first biological medicine for the treatment of inadequately controlled CRSwNP in adult patients. These authorizations were based on the results of two Phase 3 studies, SINUS-24 and SINUS-52 studies, which evaluated the effects of dupilumab administration (300 mg) every 2 weeks plus intranasal corticosteroids compared to placebo plus intranasal corticosteroids, at 24- and 52-weeks, respectively (37). According to the results of these studies, dupilumab significantly improved the signs and symptoms of severe CRSwNP. In particular, it induced improvements in nasal polyp size, sinus opacification and health-related quality of life (HR-QOL). The major symptoms of CRSwNP, including nasal congestion or obstruction, nasal discharge and loss of smell, were relieved. Moreover, it allowed a reduction in the use of systemic corticosteroids and nasal polyp surgery, being generally well-tolerated. Furthermore, dupilumab has also been shown to improve lung function in asthmatic patients. It is important to highlight this result since many patients with CRSwNP also suffer from asthma. Recently, Laidlaw et al. reported the results of a randomized, double-blind, placebo-controlled trial according to which dupilumab improved both upper and lower airway outcome measures and HRQoL in patients with severe CRSwNP and comorbid asthma. This study also confirmed its positive tolerability profile. The most common adverse events were nasopharyngitis, headache, injection-site erythema, worsening of nasal polyposis, and asthma. These were more frequent with placebo group than dupilumab (38). 
Given the high prevalence of chronic respiratory diseases and the high cost asscociated with biological products, patient selection is crucial. During the European Forum for Research and Education in Allergy and Airway Diseases (EUFOREA) in 2019, a multidisciplinary Expert Board proposed indications for biological treatment use in CRSwNP. Some indicative criteria for biological treatment in CRSwNP patients were identified. These included evidence of type 2 inflammation with biological biomarkers, need for systemic corticosteroids (2 or more courses in the past year), significantly impaired quality of life, significant loss of smell, or diagnosis of comorbid asthma. Based on a previous history of surgery, the use of a biological treatment was suggested in patients with presence of bilateral nasal polyps if 3 or 4 aforementioned criteria are found, respectively (39). Moreover, lebrikizumab and tralokinumab are other two antibody therapeutics that prevent binding of IL-13 to its receptors. In particular, lebrikizumab targets IL-13 with high-affinity, preventing the formation of the IL-13R $\alpha 1 /$ IL- $4 \mathrm{R} \alpha$ heterodimer receptor signaling complex. Since lebrikizumab does not prevent the binding of IL-13 to the IL-13R $\alpha 2$ receptor, it does not interfere with the endogenous regulation of IL-13 (40). Actually, they have been investigated only in other pathologic conditions, in particular asthma (including allergic type) and atopic dermatitis. No trials in sinus or nasal cavities diseases have yet been conducted.

\section{Innovative Drugs for the Treatment of Oncologic Rhinological Diseases}

The nasal sinus neoplasms consist of a heterogeneous group of benign or malignant tumor histotypes which require different diagnostic-therapeutic management. Among the benign forms, the sinonasal hemangioma represents a rare vascular-type tumor of endothelial cells. Recently, a case report describing the administration of bevacizumab $(50 \mathrm{mg}$ ) as treatment for a recurrent sinonasal hemangioma has been reported in the literature. The administration was performed by intralesion injection under endoscopic visualization in a 67-year-old patient. After 10 months, a reduction in the tumor size, a complete resolution of epistaxis and nasal obstruction were observed (41). Bevacizumab is a $\mathrm{mAb}$ that, by binding the growth factor of vascular endothelial cells (VEGF), blocks its biological activity. It is indicated as treatment for several types of solid tumor. It induces regression of the tumor vascularization, inhibits the formation of new vascularization, with consequent arrest of tumor growth (42).

The major rhinologic field of application of innovative drugs is nasopharyngeal carcinoma (NPC). This is a rare type of head-neck cancer. There are $\sim 129,000$ new cases of NPC each year worldwide. Over $70 \%$ of such cases are reported in South China and Southeast Asia. This tumor is etiologically associated with the Epstein-Barr virus (EBV). It represents an "inflamed tumor" archetype, showing often a dense lymphocytic infiltrate and increased expression of the programmed death ligand (PDL1) (43). For this reason, the patients with NPC are potentially suitable for treatment with immune checkpoint inhibitors
(ICIs). The ICIs are newly introduced mAbs that have literally revolutionized the treatment of several solid tumors. Cancer cells are able to evade recognition and subsequent elimination by the immune system through a series of adaptive responses, including the overexpression of various immunosuppressive molecules in the tumor microenvironment. Some of these molecules, such as CTLA-4, PD-1, and its PD-L1, are targets of ICIs. By blocking these immunosuppressive molecules, ICIs induce the reactivation of cytotoxic $\mathrm{T}$ lymphocytes able to destroy cancer cells. ICIs treatments showed significant clinical benefit for different types of cancer, establishing immunotherapy as an important advance in cancer treatment (44). In order to evaluate the efficacy in nasopharyngeal carcinoma of some antiPD1 agents, such as pembrolizumab, nivolumab, camrelizumab, several clinical studies were conducted. Other ones are still in progress (43). Among ICIs, nivolumab has promising activity in nasopharyngeal carcinoma. Recently, the results of an international phase 2 study evaluating the antitumor activity of nivolumab in the treatment of NPC were published. This study was conducted in 44 patients with pre-treated recurrent or multiple metastatic NPC treated with nivolumab until disease progression. A complete response was observed in one patient, while eight patients showed $a \geq 30 \%$ decline in tumor dimension, defined as partial response. The disease control rate was $54.5 \%$. The 1 -year overall survival rate was $59 \%$ (95\% CI, 44.3-78.5\%) and the 1-year progressionfree survival (PFS) rate was $19.3 \%$ (95\% CI, 10.1-37.2\%) (45). Recently, the findings emerging from a first phase 2 study of ipilimumab/nivolumab combination in NPC were presented in the context of ESMO Asia Virtual Congress 2020. According to these results, this ICI combination provide durable responses in patients with recurrent or metastatic NPC (46).

\section{TECHNOLOGICAL INNOVATION}

\section{Drug-Eluting Implants}

Various types of devices are available for nasal drug delivery systems. Biomaterials and sinus implant are some of these. Thanks to the incessant progress of technology, new biomaterials and sinus implants have been investigated, providing postoperative effective local corticosteroids into the sinuses. Over the years, the biomaterials have been used in the CRS post-operative management settings. Polylactide sinus implants, polyurethane foam, and carboxymethylcellulose were commonly used biomaterials (47). The bio-absorbable implants represent an example of innovative pharmaceutical technologies. In particular, these implants allowing local release of corticosteroids (CS) could be useful in the post-operative management of endoscopic sinus surgery (ESS). In patients with CRS, even more with CRSwNP, postoperative wound healing following ESS is an important factor for procedural success. After surgical treatment, topical or systemic CS therapy, and revision surgery are the available treatment options. However, these latter have significant risks and limitations. The topical nasal CS therapy ensure more effective and lasting symptomatic benefits, as well 
as reducing the size and number of polyps and preventing polyp recurrence. However, the distribution of topical steroids in the nasal cavity and sinuses is highly variable, depending on the delivery device as well as on the anatomy of the sinus drainage pathways. Steroid-releasing bio-absorbable implants have been extensively investigated for their ability to dilate and restore patency of the sinus by local and controlled release of CS. In the literature different CS-releasing bioabsorbable implants are described. A bio-absorbable, fluticasone propionate (FP)-eluting implant (SinuBand FP) resulted well-tolerated and effective in patients with CRS and nasal polyps. In particular, the results of a first-in-human, randomized, partially doubleblind, single-tertiary-referral-center, controlled trial showed its local, and ocular safety. Compared to a standard nasal pack, or to a SinuBand without FP, SinuBand FP allowed significantly better polyp score $(p=0.03)$ and a better trend of inflammatory process. Patients receiving the bioabsorbable, fluticasone propionate-eluting implant reported lower pain (48). Moreover, bioabsorbable mometasone-eluting implants were also investigated $(49,50)$. In a prospective, randomized, doubleblinded, placebo-controlled study, the endoscopic appearance in the healing process of CRSwNP after ESS was improved in patients receiving mometasone furoate (MF)-impregnated biodegradable nasal dressings (BNDs) (51). A comprehensive, up-to-date literature review reported a novel, mometasone furoate (MF) sinus implant such as useful treatment for patients with recurrent CRSwNP after ESS, playing an important role in the management (52). MF implants were also evaluated with additional topical nasal spray therapy. According to the results of a pooled analysis of data from 2 randomized controlled trials (RCTs), it emerged that this association has allowed more favorable results, in terms of subject than objective endpoints, compared to topical therapy with nasal spray alone, being useful in the management of patients with NP, especially those who have allergic rhinitis, expanded polyposis, altered odor or ESS $<24$ months (53). So, according clinical evidences, steroid-eluting bioabsorbable implants result safe and effective in the reduction of polyp size, symptom burden, and the need for revision sinus surgery. Favorable safety profile and efficacy of bioabsorbable steroid-impregnated implants in improving the healing process following ESS emerged from a recent meta-analysis including eight randomized controlled trials (54). About $14 \%$ of CRS patients undergoing surgery require ESS revision for a variety of reasons, including recurrence of nasal polyps and inflammation, adhesion formation, middle turbinate lateralization. So, the use of the nasal bioabsorbable implants appears to have a favorable economic impact. In fact, considering the substantial annual revision ESS costs, the use of the implant instead of revision ESS could result in considerable cost savings (55). Sinus implants made up of bioabsorbable polymers represent another new method to optimize surgical outcomes and to treat recurrent nasal polyposis after ESS. They allow sustained-release corticosteroids to be delivered locally directly to inflamed sinus tissues. Once implanted, these expand to fit different sizes and shapes, adapting to the space after surgery (47).

\section{Super-Selective Intra-Arterial Infusion of Chemotherapy With Concomitant Radiotherapy}

Another innovative technological approach emerged also for the treatment of the maxillary sinus cancer (MSC). It is represented by the super-selective intra-arterial infusion of chemotherapy with concomitant radiotherapy (RADPLAT). It was developed in order to overcome some patients' problems related to advanced MSC surgical procedures, such as impaired facial function and significant facial deformity. Moreover, it is useful therapeutic strategy also for those patients with stage T4b MSC for which there is no indication of surgical resection $(56,57)$. The superselective intra-arterial chemotherapy with radiation therapy reflects a precision medicine approach associated with a low risk of side effects. This effective procedure might be useful to avoid highly invasive surgery (58). However, the published studies refer to small patient samples (56-59). The introduction of technological innovations has allowed a significant expansion of outpatient rhinology (60).

\section{Balloon Catheter Dilatation}

Innovation refers also to improved old technologies, like balloon-dilatation. Since 2005 the balloon catheter dilatation $(\mathrm{BCD})$ represents a useful intervention for the management of CRS. BCD is a minimally invasive procedure aimed to restore physiological sinus drainage, safely dilating sinuses through microfractures (61). BCD has become a common treatment for chronic sinusitis in the United States (62). It is among the most common office-based rhinological procedures (60). Over the years, it has been renewed in several aspects, including innovations in ergonomics and lighted guidewires in order to make the utilization more effective and safer. Now, the new devices are equipped with suction and irrigation capabilities and allow multisinus applications using just one device. Moreover, the previous tools used fluoroscopy for localization with the consequent risks of exposure to radiation. Today, transillumination and real-time 3-dimensional image guidance have been introduced to overcome these problems. Some studies suggest the use of $\mathrm{BCD}$ as a safe tool in the management of pediatric CRS (pCRS). However, they refer to small samples and show methodological limitations (63-65). According to recent meta-analyses and systematic reviews, more evaluations are needed to demonstrate its clinical usefulness in terms of improving the quality of life and the comparative efficacy of BCD compared to standard treatment regimens in specific patients' subgroups such as children $(61,65-67)$. Moreover, as with all interventions, $\mathrm{BCD}$, although minimally invasive, can be associated with adverse events such as cerebrospinal fluid leaks, mainly reported with frontal sinus procedures (62).

\section{Artificial Intelligence and Machine Learning}

In the era of big data, the application of artificial intelligence (AI), the machine learning (ML), and particularly deep learning, represent increasingly relevant topics of the health care research, also in the rhinology field. Recently, in order to efficiently use 
all recorded data, AI and ML technology has been used in some studies of chronic rhinitis and allergic rhinitis, providing some exciting new research modalities. Regarding the application of AI and ML technology, few reports describe their use in rhinology. Only recently (since 2015) a slow increase in their descriptions emerged in literature. In the majority of the rhinologic studies in which an AI approach was used, cluster analyses were performed, i.e., to predict surgical vs. medical treatments for CRS in patients who did not have successful outcomes after initial medical treatment (68). Regarding the ML technology, the majority of algorithms are divided into supervised or unsupervised learning. This latter has been reported as a novel tool in the investigation of CRS. It represents a paradigm shift from the traditional approach based upon the clinically recognized phenotypes of CRS "with polyps" and "without polyps." Instead, an unsupervised learning approach using the application of complex mathematical models is able to derive other different subgroups which can then be further examined (69).

\section{Augmented and Virtual Reality}

Finally, also the augmented reality (AuR) and the virtual reality (VR) represent other new technological approaches applied to the rhinology field. The main difference between these new technologies consists in an enhancement of a user's natural vision obtained with $\mathrm{AuR}$, that instead, in VR, comes completely replaced. Today, the VR can be used in the surgical simulation, allowing modernization of training and its transition from the practice of simple exercises into a fully-immersive environment experience. In a recent study, the use of a virtual coach was tried guiding a group of surgeons using surgical videos, auditory, and visual cues (70). In AuR, the real-world environments are combined with computer-generated sounds, text, and graphics. So, the AuR represents a tool for the surgeons that improve visualization, location, and orientation allowing improvement of surgical outcomes in terms of operating time, precision, and increased surgeon confidence. AuR can also represent a tool for procedure simulations or anatomy education, allowing the students to learn head and neck anatomy, often difficult to conceptualize. It has grown rapidly and continues to expand (71). Rarely, the AR has been used as a diagnostic and treatment tool through specific AuR-based platforms described in some studies.

\section{DIGITAL INNOVATION}

Today, we are in the digital era. Digital tools and devices are ubiquitous. We are learning to exploit the goals achieved in terms of connectivity and connection also for the management of health and, therefore, of diseases. Thanks to the achieved technology progress, therapeutic treatments can take advantage of software and devices. The digital approach is particularly able to obtain a real-time control and support of behavior and health status, improving quality of health care in the long term by greater patient involvement. We are witnessing to the introduction of digital therapeutics, as clinically validated treatments designed to complement or potentially replace traditional therapies (72). Moreover, digital advances have allowed innovative, almost futuristic approaches such as that of digital twins. These latter represent an engineering concept which can be applied to different complex systems, including that of human physiology (73). Digital twins are built on computer-based models that are fed individual and population data. The translation of the digital twin concept to patients aims to improve diagnostics and treatment in order to deliver data-driven personalized medicine. Beyond these revolutionary paths, digital progress is applied even to innovative approaches that are much more accessible. These take the form in eHealth, mobile-Health (mHealth) such as the telemedicine based on the obtained connectivity of mobile devices with the internet (74-77). Today, given the current COVID-19 pandemic, several professional societies are encouraging the maximization of the use of telemedicine in current practice (78). This approach introduced a new way for generating health and medical databy the individual, in real time, in a real-world environment. Although these features are interesting, the benefits of digital medicine have to be proven through rigorous research, especially validation through randomized, controlled clinical trials. The mHealth, as a branch of eHealth defined as "medical and public health practice supported by mobile devices," can be effective to facilitate communication between primary care providers, able to overcome geographical and temporal barriers as well as to treatment accessibility and availability. It can use different tools, including smartphone applications (app), SMS text messaging with a support service, physical symptom tracking through wearable technologies, and receiving virtual therapy. The mHealth tools are developed to improve patient empowerment via education and self-management and will hopefully contribute to better patient adherence, quality feedback to the physician and improved patient health literacy. As reported by mHealth users, it is advantageous compared with faceto-face therapy, allowing them to be more open and honest (79). Moreover, mHealth therapy allows rapid adaptation of the treatment strategy based on the symptoms, concomitant medications and key events that may impact the disease. In particular, mobile applications are achieving a prominent position in the management of chronic diseases. For chronic respiratory diseases, most of the apps have been developed for lower respiratory diseases such as asthma or COPD. Recently, Bodini et al. have identified 5 Digital Therapeutics (DTx) for asthma and COPD which combine sensor devices, mApps for patients, and cloud-based software for healthcare professionals (80). They consent to record if/when/how the patient uses the inhaler, to alert for use the inhaler, to receive information from the sensor, providing a personalized support and remote monitoring. To date, mySinusitisCoach is a mobile app available for patients with sinus disease (81). This tool has been launched during the European Rhinology Research Forum (ERRF) 2017. It was designed, developed and implemented to support CRS patients in monitoring their symptoms and to provide patients with a digital support platform containing reliable medical information about their disease and treatment options. MySinusitisCoach has been developed thanks to a collaboration between CRS medical experts, patients, general practitioners and community pharmacists. This collaboration was sought to obtain a tool that would meet the needs of both 
patients and healthcare professionals. Its functionalities include the monitoring of symptoms and consumption of drugs, the visualization of the disease control level, providing unbiased information on chronic sinusitis and asthma. Moreover, the easy sharing of data with the doctor in order to obtain a realtime connection between the patients and health workers, allows optimization of treatment. Recently, a cross-sectional evaluation of data obtained by users of mySinusitisCoach. This real-life assessment confirms the high disease burden in uncontrolled CRS patients, which can be supported by mobile technology in the real-life monitoring (82). The mobile apps allows not only a continuous and remote monitoring of the patient's health status, but also an important collection of real-world data that will help in clinical studies validating patient stratification as well as understanding of the socio-economic impact of CRS, in order to improve treatment strategies. Recently, mySinusitisCoach has been replaced by Galenus Health, a mobile app developed by a team of internationally recognized doctors designed for anyone with asthma, respiratory allergy or chronic sinusitis, often concomitant diseases. Finally, the use of digital approach with a smart language can also be used for the improvement of patient education. In fact, its use can positively impact on patient outcomes such as anxiety, pain and satisfaction in relation to the perioperative patient experience. Online education materials are often too complex, inaccurate or misleading to be useful to the patient. A recent study has been conducted by University of British Columbia in order to evaluate the effect of patient education videos on perioperative anxiety in patients undergoing endoscopic sinus surgery. The enrolled patients received four short YouTube videos explaining chronic rhinosinusitis and endoscopic sinus surgery. Patients of the control group received the standard of care patient education with verbal and written education. The study is completed but results not yet available $(83,84)$.

\section{CONCLUSION}

Innovative aspects in rhinology involve new drugs, technologies for their administration as well as digital applications. Each different innovative tool has an important impact and allows an improvement in several clinical and patients outcomes, including quality of life, efficacy and safety. The discussed innovative tools show an overlapping nature among the considered fields (Figure 3). The emerging innovative drugs include mAbs targeting on characteristic pathways of type 2 inflammation, such as those of IgE, the IL-5, and IL-4/IL-13 which are involved in several pathologic condictions including CRSwNP or allergic rhinitis. Dupilumab (anti IL-4), mepolizumab (anti IL-5), and omalizumab (antiIgE) represent the main mAbs developed such as innovative treatment options for patients with NP and CRS. They seem to allow improvement in terms of quality of life, disease severity and tolerability of treatment. Other mAbs are in the advanced research stages like etokimab (anti-IL33) or tezepelumab (targeting on TSLP). Moreover, another important application field of mAbs is the oncological immunotherapy. Considering the higher expression of PD-L1 in NPC, the use

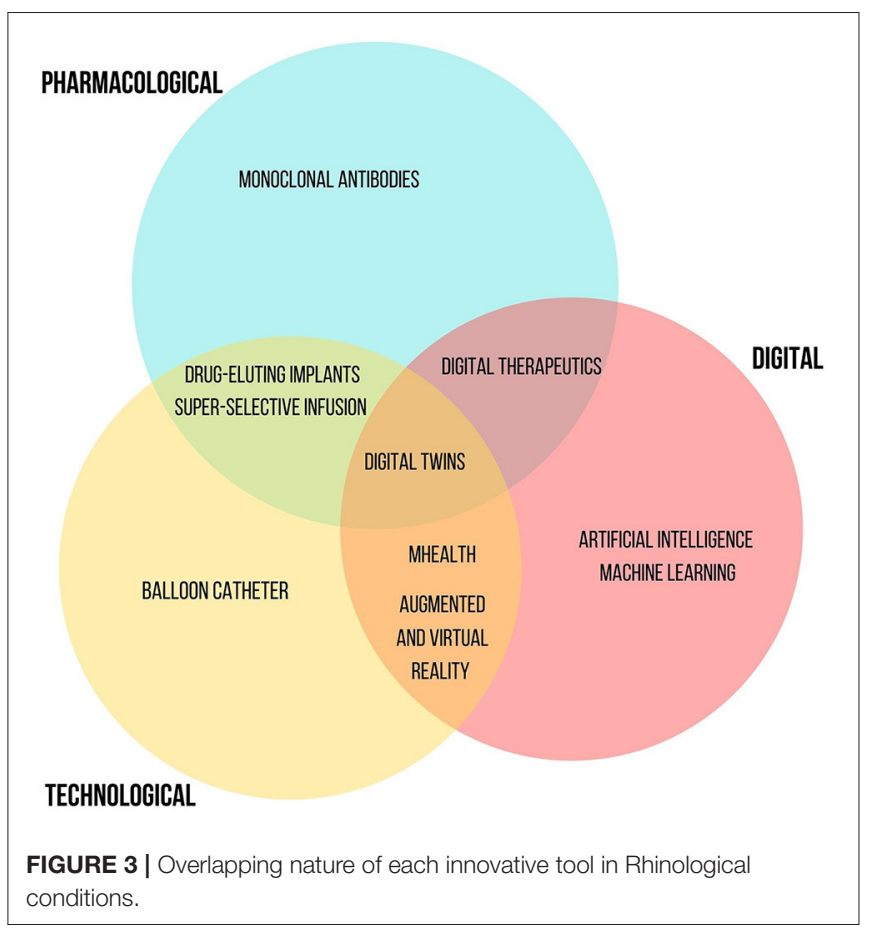

of PD-1 inhibitors, such as nivolumab, or a dual CTLA-4/PD1 blockade (85) appear to be an effective strategy for the treatment of this cancer form. However, current studies are not yet at in advanced stages. The careful monitoring of patients with regard to the autoimmune toxicity related to them should not be underestimated. Regarding the technological innovation, the implants with bio-absorbable biomaterials represent new interesting available technologies. In particular, those allowing the topical administration of corticosteroids drugs (fluticasone and mometasone) are useful treatments for patients with recurrent CRSwNP after ESS, playing an important role in its management. Moreover, considering the substantial annual costs of ESS, their favorable economic impact is worthy of note. Advanced technologies such as, AI and ML, as well as AuR and VR have also proved useful in the rhinologic field with their main impacts on precision medicine and surgery. Finally, the development and use of mHealth tools represent a winning strategy in monitoring therapy success, safety and tolerability as well as the progress of chronic disease including CRSwNP. They seem to be efficient and effective mainly in the improvement of patients' outcomes. The mobile apps allow to improve patient empowerment by an active participation in the decision-making process of the therapeutic plan. Likewise, their use allows collection of real-world data that will help to improve treatment strategies in a greater perspective of personalized and precision medicine. So, supporting the research of innovative tools and strategies (including pharmacological, technologic, or digital ones) is essential to improve the management of chronic diseases that significantly affect the quality of life of patients. Further studies are strongly needed in order to support their use 
in real life context. In the future, the use of combined innovative approaches is desirable.

\section{AUTHOR CONTRIBUTIONS}

FR, AC, and RR: drafting the work and revising it for important intellectual content. RR, GioM, and GiuM: substantial contributions to the acquisition, analysis, or interpretation of data for the work. FR, CR, and ADC:

\section{REFERENCES}

1. Harrison TW, Chanez P, Menzella F, Canonica GW, Louis R, Cosio BG, et al. Onset of effect and impact on health-related quality of life, exacerbation rate, lung function, and nasal polyposis symptoms for patients with severe eosinophilic asthma treated with benralizumab (ANDHI): a randomised, controlled, phase 3b trial. Lancet Respir Med. (2021) 9:26074. doi: 10.1016/S2213-2600(20)30414-8

2. Scavone C, di Mauro G, Mascolo A, Berrino L, Rossi F, Capuano A. The new paradigms in clinical research: from early access programs to the novel therapeutic approaches for unmet medical needs. Front Pharmacol. (2019) 10:111. doi: 10.3389/fphar.2019.00111

3. Aifa. Allegato 1 Criteri Per La Valutazione Dellinnovativita'. (2021). Available online at: http://www.jclinepi.com/content/jce-GRADE-Series (accessed April 8, 2021).

4. del Nazareno L. Farmindustria Centro Studi. (2021). Available online at: www.farmindustria.it (accessed April 9, 2021).

5. Scavone C, Rafaniello C, Berrino L, Rossi F, Capuano A. Strengths, weaknesses and future challenges of biosimilars' development. An opinion on how to improve the knowledge and use of biosimilars in clinical practice. Pharmacol Res. (2017) 126:138-42. doi: 10.1016/j.phrs.2017.11.002

6. Mahler S. Safety of biologics therapy: monoclonal antibodies, cytokines, fusion proteins, hormones, enzymes, coagulation proteins, vaccines, botulinum toxins. MAbs. (2017) 9:885-8. doi: 10.1080/19420862.2017. 1343709

7. Lindsley CW. Predictions and statistics for the best-selling drugs globally and in the United States in 2018 and a look forward to 2024 projections. ACS Chem Neurosci. (2019) 10:1115. doi: 10.1021/acschemneuro.9b00112

8. Scavone C, Brusco S, Bertini M, Sportiello L, Rafaniello C, Zoccoli A, et al. Current pharmacological treatments for COVID-19: what's next? Br. J Pharmacol. (2020) 177:4813-24. doi: 10.1111/bph.15072

9. Scavone C, Mascolo A, Rafaniello C, Sportiello L, Trama U, Zoccoli A, et al. Therapeutic strategies to fight COVID-19: which is the status artis? $\mathrm{Br} \mathrm{J}$ Pharmacol. (2021). 2021:15452. doi: 10.1111/bph.15452

10. Gevaert P, Calus L, Van Zele T, Blomme K, De Ruyck N, Bauters $\mathrm{W}$, et al. Omalizumab is effective in allergic and nonallergic patients with nasal polyps and asthma. J Allergy Clin Immunol. (2013) 131:47. doi: 10.1016/j.jaci.2012.07.047

11. Annunziato F, Romagnani C, Romagnani S. The 3 major types of innate and adaptive cell-mediated effector immunity. J Allergy Clin Immunol. (2015) 135:626-35. doi: 10.1016/j.jaci.2014.11.001

12. Lyly A, Laulajainen-Hongisto A, Gevaert P, Kauppi P, Toppila-Salmi S. Monoclonal antibodies and airway diseases. Int J Mol Sci. (2020) 21:121. doi: 10.3390/ijms21249477

13. Avdeeva K, Fokkens W. Precision medicine in chronic rhinosinusitis with nasal polyps. Curr Allergy Asthma Rep. (2018) 18. doi: $10.1007 /$ s11882-018-0776-8

14. Dietz de Loos D, Lourijsen ES, Wildeman MAM, Freling NJM, Wolvers MDJ, Reitsma S, et al. Prevalence of chronic rhinosinusitis in the general population based on sinus radiology and symptomatology. J Allergy Clin Immunol. (2019) 143:1207-14. doi: 10.1016/j.jaci.2018.12.986

15. Lee K, Tai J, Lee SH, Kim TH. Advances in the knowledge of the underlying airway remodeling mechanisms in chronic rhinosinusitis based on the endotypes: a review. Int J Mol Sci. (2021) 22:1-16. doi: 10.3390/ijms22020910 final approval of the version to be published. $\mathrm{AC}, \mathrm{AD}$, and GaM: agreement to be accountable for all aspects of the work in ensuring that questions related to the accuracy or integrity of any part of the work are appropriately investigated and resolved. FR and GaM: developed the concept and designed the study. RR and GioM: wrote the paper. All authors contributed to the article and approved the submitted version.

16. Chong LY, Piromchai P, Sharp S, Snidvongs K, Webster KE, Philpott C, et al. Biologics for chronic rhinosinusitis. Cochrane Database Syst Rev. (2021) 2021:CD013513. doi: 10.1002/14651858.CD013513.pub3

17. Bidder T, Sahota J, Rennie C, Lund VJ, Robinson DS, Kariyawasam HH. Omalizumab treats chronic rhinosinusitis with nasal polyps and asthma together-a real life study. Rhinol J. (2018) 56:42-5. doi: 10.4193/Rhin17.139

18. Delemarre T, Holtappels G, De Ruyck N, Zhang N, Nauwynck H, Bachert $\mathrm{C}$, et al. Type 2 inflammation in chronic rhinosinusitis without nasal polyps: another relevant endotype. J Allergy Clin Immunol. (2020) 146:33743.e6. doi: 10.1016/j.jaci.2020.04.040

19. Fokkens WJ, Lund VJ, Hopkins C, Hellings PW, Kern R, Reitsma S, et al. European position paper on rhinosinusitis and nasal polyps 2020. Rhinology. (2020) 58:1-464. doi: 10.4193/Rhin20.401

20. Tsetsos N, Goudakos JK, Daskalakis D, Konstantinidis I, Markou K. Monoclonal antibodies for the treatment of chronic rhinosinusitis with nasal polyposis: a systematic review. Rhinol J. (2018) 56:11-21. doi: 10.4193/Rhin 17.156

21. Fahy JV. Type 2 inflammation in asthma-present in most, absent in many. Nat Rev Immunol. (2015) 15:57-65. doi: 10.1038/nri3807

22. Meier EC, Schmid-Grendelmeier P, Steiner UC, Soyka MB. Reallife experience of monoclonal antibody treatments in chronic rhinosinusitis with nasal polyposis. Int Arch Allergy Immunol. (2021) 2021:1-8. doi: 10.1159/000514262

23. Chen Y, Wang W, Yuan H, Li Y, Lv Z, Cui Y, et al. Current state of monoclonal antibody therapy for allergic diseases. Engineering. (2021) 6:29. doi: 10.1016/j.eng.2020.06.029

24. Schafflick D, Kieseier BC, Wiendl H, Meyer zu Horste G. Novel pathomechanisms in inflammatory neuropathies. J Neuroinflammation. (2017) 14:8. doi: 10.1186/s12974-017-1001-8

25. Nagase H, Ueki S, Fujieda S. The roles of IL-5 and anti-IL-5 treatment in eosinophilic diseases: asthma, eosinophilic granulomatosis with polyangiitis, and eosinophilic chronic rhinosinusitis. Allergol Int. (2020) 69:17886. doi: 10.1016/j.alit.2020.02.002

26. Gevaert P, Van Bruaene N, Cattaert T, Van Steen K, Van Zele T, Acke F, et al. Mepolizumab, a humanized anti-IL-5 mAb, as a treatment option for severe nasal polyposis. J Allergy Clin Immunol. (2011) 128:989-5.e18. doi: 10.1016/j.jaci.2011.07.056

27. Rivero A, Liang J. Anti-IgE and anti-IL5 biologic therapy in the treatment of nasal polyposis: a systematic review and meta-analysis. Ann Otol Rhinol Laryngol. (2017) 126:739-47. doi: 10.1177/0003489417731782

28. Roufosse F. Targeting the interleukin-5 pathway for treatment of eosinophilic conditions other than asthma. Front Med. (2018) 5:1. doi: 10.3389/fmed.2018.00049

29. EMA. Assessment Report on Extension(S) of Marketing Authorisation Nucala. (2021). Available online at: www.ema.europa.eu/contact (accessed April 28, 2021).

30. Bachert C, Sousa AR, Lund VJ, Scadding GK, Gevaert P, Nasser $\mathrm{S}$, et al. Reduced need for surgery in severe nasal polyposis with mepolizumab: randomized trial. J Allergy Clin Immunol. (2017) 140:102431.e14. doi: 10.1016/j.jaci.2017.05.044

31. Han JK, Bachert C, Fokkens W, Desrosiers M, Wagenmann M, Lee SE, et al. Mepolizumab for chronic rhinosinusitis with nasal polyps (SYNAPSE): a randomised, double-blind, placebo-controlled, phase 3 trial. Lancet Respir Med. (2021) 97:7. doi: 10.1016/S2213-2600(21)00097-7 
32. Markham A. Reslizumab: first global approval. Drugs. (2016) 76:90711. doi: $10.1007 / s 40265-016-0583-2$

33. Cardet JC, Israel E. Update on reslizumab for eosinophilic asthma. Expert Opin Biol Ther. (2015) 15:1531. doi: 10.1517/14712598.2015.1090972

34. ClinicalTrials.gov. Effect of Reslizumab in Chronic Rhinosinusitis. ClinicalTrials.gov. (2021). Available online at: https://clinicaltrials.gov/ct2/ show/NCT02799446? intr=Resliumab+OR+sch+55700anddraw=3andrank= 14 (accessed April 28, 2021).

35. Vlastos I, Gkouskou K, Doulaptsi M, Karatzanis A, Prokopakis EP. Precision medicine in rhinosinusitis. Curr Allergy Asthma Rep. (2019) 19. doi: $10.1007 / \mathrm{s} 11882-019-0850-\mathrm{x}$

36. Tversky J, Lane AP, Azar A. Benralizumab effect on severe chronic rhinosinusitis with nasal polyps (CRSwNP): a randomized double-blind placebo-controlled trial. Clin Exp Allergy. (2021) 2021:cea.13852. doi: 10.1111/cea.13852

37. Bachert C, Han JK, Desrosiers M, Hellings PW, Amin N, Lee SE, et al. Efficacy and safety of dupilumab in patients with severe chronic rhinosinusitis with nasal polyps (LIBERTY NP SINUS-24 and LIBERTY NP SINUS-52): results from two multicentre, randomised, double-blind, placebo-controlled, parallel-group phase 3 trials. Lancet. (2019) 394:163850. doi: 10.1016/S0140-6736(19)31881-1

38. Laidlaw TM, Bachert C, Amin N, Desrosiers M, Hellings PW, Mullol J, et al. Dupilumab improves upper and lower airway disease control in chronic rhinosinusitis with nasal polyps and asthma. Ann Allergy Asthma Immunol. (2021) 1:12. doi: 10.1016/j.anai.2021.01.012

39. Fokkens WJ, Lund V, Bachert C, Mullol J, Bjermer L, Bousquet J, et al. EUFOREA consensus on biologics for CRSwNP with or without asthma. Allergy. (2019) 74:2312-9. doi: 10.1111/all.13875

40. Guttman-Yassky E, Blauvelt A, Eichenfield LF, Paller AS, Armstrong AW, Drew J, et al. Efficacy and safety of lebrikizumab, a high-affinity interleukin 13 inhibitor, in adults with moderate to severe atopic dermatitis: a phase 2b randomized clinical trial. J Am Med Assoc Dermatol. (2020) 156:41120. doi: 10.1001/jamadermatol.2020.0079

41. Kinzinger MR, Strong EB, Bernard J, Steele TO. Intralesional bevacizumab for the treatment of recurrent sinonasal hemangioma. Ann Otol Rhinol Laryngol. (2018) 127:969-73. doi: 10.1177/0003489418802288

42. Garcia J, Hurwitz HI, Sandler AB, Miles D, Coleman RL, Deurloo R, et al. Bevacizumab (Avastin ${ }^{\circledR}$ ) in cancer treatment: a review of 15 years of clinical experience and future outlook. Cancer Treat Rev. (2020) 86. doi: 10.1016/j.ctrv.2020.102017

43. Chen YP, Chan ATC, Le QT, Blanchard P, Sun Y, Ma J. Nasopharyngeal carcinoma. Lancet. (2019) 394:64-80. doi: 10.1016/S0140-6736(19)30956-0

44. Ruggiero R, Fraenza F, Scavone C, di Mauro G, Piscitelli R, Mascolo A, et al. Immune checkpoint inhibitors and immune-related adverse drug reactions: data from Italian Pharmacovigilance Database. Front Pharmacol. (2020) 11:1. doi: 10.3389/fphar.2020.00830

45. Ma BBY, Lim WT, Goh BC, Hui EP, Lo KW, Pettinger A, et al. Antitumor activity of nivolumab in recurrent and metastatic nasopharyngeal carcinoma: an international, multicenter study of the mayo clinic phase 2 consortium (NCI-9742). J Clin Oncol. (2018) 388:1412-8. doi: 10.1200/JCO.2017.77.0388

46. Kao H-F, Ang M-K, Ng QS, et al. Combination ipilimumab and nivolumab in recurrent/metastatic nasopharyngeal carcinoma (R/M NPC) Ü updated efficacy and safety analysis of NCT03097939. ESMO Asia Vir Cong. (2020).

47. Tai J, Lee K, Kim TH. Pharmaceutics current perspective on nasal delivery systems for chronic rhinosinusitis. Pharmaceutics. (2021) 13:246. doi: 10.3390/pharmaceutics 13020246

48. Adriaensen GFJPM, Lim KH, Fokkens WJ. Safety and efficacy of a bioabsorbable fluticasone propionate-eluting sinus dressing in postoperative management of endoscopic sinus surgery: a randomized clinical trial. Int Forum Allergy Rhinol. (2017) 7:813-20. doi: 10.1002/alr.21963

49. Han JK, Forwith KD, Smith TL, Kern RC, Brown WJ, Miller SK, et al. RESOLVE: a randomized, controlled, blinded study of bioabsorbable steroideluting sinus implants for in-office treatment of recurrent sinonasal polyposis. Int Forum Allergy Rhinol. (2014) 4:861-70. doi: 10.1002/alr.21426

50. Forwith KD, Han JK, Stolovitzky JP, Yen DM, Chandra RK, Karanfilov B, et al. RESOLVE: bioabsorbable steroid-eluting sinus implants for in-office treatment of recurrent sinonasal polyposis after sinus surgery: 6-month outcomes from a randomized, controlled, blinded study. Int Forum Allergy Rhinol. (2016) 6:573-81. doi: 10.1002/alr.21741

51. Zhao KQ, Yu YQ, Yu HM. Effects of mometasone furoate-impregnated biodegradable nasal dressing on endoscopic appearance in healing process following endoscopic sinus surgery: a randomized, doubleblind, placebo-controlled study. Int Forum Allergy Rhinol. (2018) 8:1233-41. doi: 10.1002/alr.22213

52. Shen J, Welch K, Kern R. Mometasone furoate sinus implant-a new targeted approach to treating recurrent nasal polyp disease. Expert Rev Clin Pharmacol. (2018) 11:1163-70. doi: 10.1080/17512433.2018.154 9485

53. Stolovitzky JP, Kern RC, Han JK, Forwith KD, Ow RA, Wright SK, et al. In-office placement of mometasone furoate sinus implants for recurrent nasal polyps: a pooled analysis. Am J Rhinol Allergy. (2019) 33:54558. doi: 10.1177/1945892419850924

54. Li W, Lu H, Wang H, Sun X, Wang D. Efficacy and safety of steroidimpregnated implants following sinus surgery: a meta-analysis. Laryngoscope. (2020) 130:2754-9. doi: 10.1002/lary.28388

55. Ernst FR, Imhoff RJ, DeConde A, Manes RP. Budget impact of a steroideluting sinus implant versus sinus surgery for adult chronic sinusitis patients with nasal polyps. I Manag Care Spec Pharm. (2019) 25:94150. doi: $10.18553 /$ jmcp. 2019.18285

56. Homma A, Sakashita T, Yoshida D, Onimaru R, Tsuchiya K, Suzuki F, et al. Superselective intra-arterial cisplatin infusion and concomitant radiotherapy for maxillary sinus cancer. Br J Cancer. (2013) 109:29806. doi: 10.1038/bjc.2013.663

57. Ashida $\mathrm{H}$, Igarashi $\mathrm{T}$, Nozawa $\mathrm{Y}$, Munetomo $\mathrm{Y}$, Higuchi T, Yamauchi $\mathrm{H}$, et al. Imaging predictor of ophthalmic involvement in maxillary sinus cancer during super selective intra-arterial cisplatin infusion and concomitant radiotherapy (RADPLAT). Head Face Med. (2021) 17:285. doi: 10.1186/s13005-021-00285-z

58. Makino T, Tachibana T, Kariya S, Matsui Y, Matsuzak I, Fujimoto S, et al. Pathological evaluation of radiotherapy and concomitant intraarterial cisplatin for maxillary sinus cancer. Auris Nasus Larynx. (2020) 47:8816. doi: 10.1016/j.anl.2020.04.004

59. Ebara T, Ando K, Eishima J, Suzuki M, Kawakami T, Horikoshi H, et al. Radiation with concomitant superselective intra-arterial cisplatin infusion for maxillary sinus squamous cell carcinoma. Jpn J Radiol. (2019) 37:4949. doi: 10.1007/s11604-019-00827-1

60. Lee JT, DelGaudio J, Orlandi RR. Practice patterns in office-based rhinology: survey of the American Rhinologic Society. Am J Rhinol Allergy. (2019) 33:26-35. doi: 10.1177/1945892418804904

61. Minni A, Dragonettp A, Sciuto A, Cavaliere C, Rosati D, Azimonti D, et al. Use of balloon catheter dilation vs. traditional endoscopic sinus surgery in management of light and severe chronic rhinosinusitis of the frontal sinus: a multicenter prospective randomized study. Eur Rev Med Pharmacol Sci. (2018) 22:285-93. doi: 10.26355/eurrev_ 201801_14170

62. Wright A, Davis E, Khan M, Chaaban M. Exploring balloon sinuplasty adverse events with the innovative openFDA database. Am J Rhinol Allergy. (2020) 34:626-31. doi: 10.1177/1945892420920505

63. Ramadan H, Terrell A. Balloon catheter sinuplasty and adenoidectomy in children with chronic rhinosinusitis. Ann Otol Rhinol Laryngol. (2010) 119:578-82. doi: 10.1177/000348941011900902

64. Gerber M, Kennedy A. Adenoidectomy with balloon catheter sinuplasty: a randomized trial for pediatric rhinosinusitis. Laryngoscope. (2018) 128:28937. doi: 10.1002/lary.27270

65. Zalzal H, Makary C, Ramadan H. Long-term effectiveness of balloon catheter sinuplasty in pediatric chronic maxillary sinusitis. Ear Nose Throat J. (2019) 98:207-11. doi: 10.1177/0145561319840126

66. Levy JM, Marino MJ, McCoul ED. Paranasal sinus balloon catheter dilation for treatment of chronic rhinosinusitis: a systematic review and meta-analysis. Otolaryngol. Head Neck Surg. (2016) 154:33-40. doi: 10.1177/0194599815613087

67. Patel V, O'Brien D, Ramadan J, Carr M. Balloon catheter dilation in pediatric chronic rhinosinusitis: a meta-analysis. Am J Rhinol Allergy. (2020) 34:694702. doi: $10.1177 / 1945892420917313$ 
68. Jun Y, Jung J, Lee H. Medical data science in rhinology: background and implications for clinicians. Am J Otolaryngol. (2020) 41:102627. doi: 10.1016/j.amjoto.2020.102627

69. Walker A, Surda P. Unsupervised learning techniques for the investigation of chronic rhinosinusitis. Ann Otol Rhinol Laryngol. (2021) 128:11706. doi: 10.1177/0003489419863822

70. Richards JP, Done AJ, Barber SR, Jain S, Son Y-J, Chang EH. Virtual coach: the next tool in functional endoscopic sinus surgery education. Int Forum Allergy Rhinol. (2020) 10:97-102. doi: 10.1002/alr.22452

71. Wong K, Yee HM, Xavier BA, Grillone GA. Applications of augmented reality in otolaryngology: a systematic review. Otolaryngol Head Neck Surg. (2018) 159:956-67. doi: 10.1177/0194599818796476

72. Waltz E. First video game to treat disease gains FDA okay. Nat Biotechnol. (2020) 38:1224-5. doi: 10.1038/s41587-020-0726-6

73. Chen X, Feng Y, Zhong W, Kleinstreuer C. Numerical investigation of the interaction, transport and deposition of multicomponent droplets in a simple mouth-throat model. J Aerosol Sci. (2017) 105:108-27. doi: 10.1016/j.jaerosci.2016.12.001

74. Bentvelsen RG, Holten E, Chavannes NH, Veldkamp KE. eHealth for the prevention of healthcare-associated infections: a scoping review. J Hosp Infect. (2021) 4:29. doi: 10.1016/j.jhin.2021.04.029

75. Kern-Goldberger AR, Haeri S, Lindsley W, Srinivas SK. Examining ultrasound diagnostic performance improvement with utilization of maternal-fetal medicine tele-interpretation. Am J Obstet Gynecol. (2021) 2021:100389. doi: 10.1016/j.ajogmf.2021.100389

76. McLoughlin L, Carey C, Dooley S, Kennedy H, McLoughlin I. An observational study of a cross platform risk assessment mobile application in a forensic inpatient setting. J Psychiatr Res. (2021) 138:388-92. doi: 10.1016/j.jpsychires.2021.04.034

77. Proulx-Cabana S, Segal TY, Gregorowski A, Hargreaves D, Flannery H. Virtual consultations: young people and their parents' experience. Adolesc Health Med Ther Volume. (2021) 12:37-43. doi: 10.2147/AHMT.S292977

78. Corbetta-Rastelli CM, Morgan TK, Homaifar N, Deangelis L, Autry AM. Experiences in electronic consultation (eConsult) service in gynecology from a quaternary academic medical center. J Med Syst. (2021) 45:9. doi: 10.1007/s10916-021-01732-9

79. Cliffe B, Tingley J, Greenhalgh I, Stallard P. mHealth interventions for self-harm: a scoping review. J Med Internet Res. (2020) 23:25140. doi: $10.2196 /$ preprints. 25140

80. Bodini R, Grinovero M, Corsico A, Marvisi M, Recchia GG, D'Antonio S, et al. Digital therapy in the treatment of asthma and COPD - epidemiology of development and use of an emerging health technology in Respiratory Medicine. Eur Respiratory J. (2019) 2019:PA735. doi: 10.1183/13993003.congress-2019. PA735

81. Seys SF, Bousquet J, Bachert C, Fokkens WJ, Agache I, Bernal-Sprekelsen M, et al. Mysinusitiscoach: patient empowerment in chronic rhinosinusitis using mobile technology. Rhinology. (2018) 56:209-15. doi: 10.4193/Rhin17.253

82. Seys SF, De Bont S, Fokkens WJ, Bachert C, Alobid I, Bernal-Sprekelsen $\mathrm{M}$, et al. Real-life assessment of chronic rhinosinusitis patients using mobile technology: the mySinusitisCoach project by EUFOREA. Allergy Eur J Allergy Clin Immunol. (2020) 75:2867-78. doi: 10.1111/all.14408

83. ClinicalTrials.gov. Clinical Trial on Chronic Rhinosinusitis (Diagnosis): Video Education, Control - Clinical Trials Registry - ICH GCP. (2021). Available online at: https://ichgcp.net/clinical-trials-registry/NCT04595461 (accessed May 21, 2021).

84. ClinicalTrials.gov. Effect of Patient Education Videos on Perioperative Anxiety in Patients Undergoing Endoscopic Sinus Surgery - Full Text View ClinicalTrials.gov. (2021). Available online at: https://www.clinicaltrials.gov/ ct2/show/NCT04595461 (accessed May 21, 2021).

85. Ipilimumab and Nivolumab. Ipilimumab in Combination with Nivolumab Is Active in Recurrent. (2021). Available online at: https://www.esmo.org/ oncology-news/ipilimumab-in-combination-with-nivolumab-is-active-inrecurrent-and-or-metastatic-nasopharyngeal-carcinoma (accessed May 21, 2021).

Conflict of Interest: The authors declare that the research was conducted in the absence of any commercial or financial relationships that could be construed as a potential conflict of interest.

Publisher's Note: All claims expressed in this article are solely those of the authors and do not necessarily represent those of their affiliated organizations, or those of the publisher, the editors and the reviewers. Any product that may be evaluated in this article, or claim that may be made by its manufacturer, is not guaranteed or endorsed by the publisher.

Copyright (C) 2021 Ruggiero, Motta, Massaro, Rafaniello, Della Corte, De Angelis, Capuano, Motta and Rossi. This is an open-access article distributed under the terms of the Creative Commons Attribution License (CC BY). The use, distribution or reproduction in other forums is permitted, provided the original author(s) and the copyright owner(s) are credited and that the original publication in this journal is cited, in accordance with accepted academic practice. No use, distribution or reproduction is permitted which does not comply with these terms. 